\title{
Modeling Knowledge Bases for Automated Decision Making Systems - A Literature Review
}

\author{
Franz Felix Füssl, Detlef Streitferdt \\ Institute for Computer and Systems Engineering \\ Technische Universität Ilmenau \\ Ilmenau, Germany
}

\author{
Anne Triebel \\ Institute for Business Information Systems Engineering \\ Technische Universität Ilmenau \\ Ilmenau, Germany
}

\begin{abstract}
Developing automated decision making systems means dealing with knowledge in every possible manner. One of the most important points of developing artificial intelligent systems is developing a precise knowledge base with integrating self-learning mechanisms. Moreover using knowledge in expert systems or decision support systems it is necessary to document knowledge and make it visible for managing it. Main goal of this work is finding a suitable solution for modeling knowledge bases in automated decision making systems concerning both illustrating specific knowledge and learning mechanisms. There are a lot of different terms describing this kind of research, such as knowledge modeling, knowledge engineering or ontology engineering. For that reason this paper provides a comparison of the technical terms in this domain by illustrating similarities, specifics and how they are used in literature.
\end{abstract}

Keywords-Knowledge Base; Knowledge Modeling; Knowledge Engineering; Ontology Engineering; Artificial Neural Network; Expert System

\section{INTRODUCTION}

An automated decision making system (ADMS) is software that is used to make decisions application-specific and mostly autonomous. Based on rules and a consistent knowledge base such a system is able to provide solutions for different issues. The underlying knowledge is one of the most significant components and has to be modeled in a specific way. There are different options for modeling the knowledge base, especially for ADMS in the topic of large software development projects. Regarding to [1] it is necessary to identify ADMS for software development projects and in that context to reveal existing knowledge models. Therefor a comprehensive literature review according to Webster and Watson [2] will be conducted. Moreover the terms 'modeling knowledge' and 'automated decision making system' will be analyzed for distinction.

The paper addresses concretely an analysis of literature about modeling knowledge bases for ADMS that could be used for choosing toolsets in large software development projects.

As methodological approach a literature review according to Webster and Watson was selected. It can be used as basis for researching and analyzing current standards of information technology issues. Webster and Watson [2] categorize two intentions for literature reviews. For one thing it serves to specify or extend a given topic by reference to existing publications. Otherwise it can be used for developing new concepts or models by analyzing theoretical principles and previous works. Accordingly this paper presents a literature review with the first described intention about modeling knowledge bases for ADMS concerning toolsets for large software development projects. First of all the concepts or rather the keywords based on the issue to be edited have to be determined. A simple mind map is very suitable for this and will be pictured. When all relevant literatures are selected by forwards and backwards searches, the concept-matrix will demonstrate all publications identified and their affiliation to the concepts. Subsequently the topic can be analyzed and important facts will have to be presented.

This document is structured as follows: After the introduction the procedure of identifying relevant literatures are detailed in chapter II. The chapter describes the used publication databases and the searched keywords. TABLE I. contains the identified literature combined with their connection to the settled concepts and summarizes the research findings. Chapter III shows different definitions of the authors about the concepts and centralizes the important results of the literature review. Finally a conclusion is displayed with main facts of the analysis and an outline for further investigations.

\section{IDENTIFYING OF LITERATURE}

The main facts of the analysis to be created are due to the topic 'modeling knowledge bases for automated decision making systems'. Webster and Watson [2] describe the concepts as segments of the main issue with partially similar meaning or completely various facets. The authors of this paper concretized the concepts through brainstorming as Fig. 1 shows.

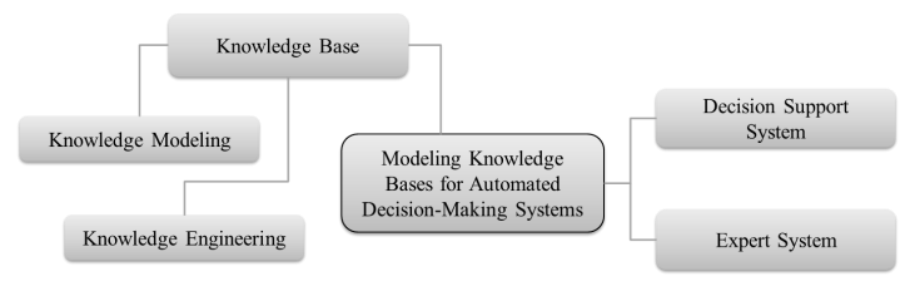

Fig. 1. Mindmap about relevant items

'Knowledge base' is deemed to be a superordinate term for knowledge modeling and knowledge engineering and will not be used for this research. The specific year range for the publications is defined from 2000 to 2015 with focus on the last 5 years based on the importance of actuality of 
contributions. Another reason is to generate a restrictive number of significant publications for analyzing. The following publication databases were used for identifying literatures due to the settled concepts: ACM Digital Library, AIS Electronic Library, EBSCO, ELSEVIER and IEEE Xplore Digital Library. In total the research aggregates to 58 papers. After examining for relevance there are 26 publications for analyzing the topic to be edited.

TABLE I. shows the concept matrix with the settled keywords and the leading authors who discuss the topics. The certain fields of application will not be displayed in this matrix but the following chapter will constitute the different range of subjects.

TABLE I. CONCEPT MATRIX

\begin{tabular}{|c|c|c|c|c|}
\hline References & 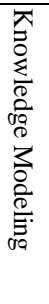 & 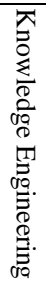 & 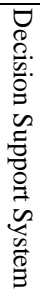 & 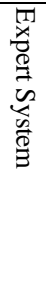 \\
\hline Chan (2002) & $\mathrm{x}$ & $\mathrm{x}$ & & $\mathrm{x}$ \\
\hline Colloc et al. (2000) & & & $\mathrm{x}$ & \\
\hline Gerards et al. (2011) & & & & $\mathrm{x}$ \\
\hline Ghoniemy et al. (2013) & & $\mathrm{x}$ & & $\mathrm{x}$ \\
\hline Guaglianone et al. (2011) & & $\mathrm{x}$ & & \\
\hline Guan et al. (2012) & & & & $\mathrm{x}$ \\
\hline Guo et al. (2008) & & & & $\mathrm{x}$ \\
\hline Hall (2012) & & $\mathrm{x}$ & & \\
\hline Hwang et al. (2011) & & & & $\mathrm{x}$ \\
\hline Kulinich (2012) & & & & $\mathrm{x}$ \\
\hline Liu et al. (2010) & $\mathrm{x}$ & & & $\mathrm{x}$ \\
\hline Lu et al. (2001) & & & $\mathrm{x}$ & \\
\hline Mendis et al. (2007) & $\mathrm{x}$ & & & \\
\hline Mistrik et al. (2014) & & $\mathrm{x}$ & & \\
\hline Moawad et al. (2013) & & $\mathrm{x}$ & & \\
\hline Nguyen et al. (2000) & & & & $\mathrm{x}$ \\
\hline Oguntimilehin et al. (2014) & & & $\mathrm{x}$ & \\
\hline Paul et al. (2012) & & & $\mathrm{x}$ & \\
\hline Popescu et al. (2014) & & & $\mathrm{x}$ & \\
\hline Prat et al. (2010) & & & $\mathrm{x}$ & \\
\hline Ramirez et al. (2011) & $\mathrm{x}$ & & & \\
\hline Sheng et al. (2010) & & & $\mathrm{x}$ & \\
\hline \multicolumn{5}{|l|}{ Tang (2014) } \\
\hline Wang et al. (2011) & & & & $\mathrm{x}$ \\
\hline Wu et al. (2015) & & $\mathrm{x}$ & & \\
\hline
\end{tabular}

The table displays the occurrence of the concepts dependent on the combination of the terms within the research. Expert system (ES) and decision support system (DSS) are established keywords that will be often used in publication databases. Knowledge engineering or knowledge modeling combined with DSS or ES were less found in articles as shown in the concept matrix. The keyword 'knowledge modeling' is currently used very rare in combination with an ADMS. It could indicate that knowledge modeling is rather called knowledge engineering and it could probably mean the same. The research implies that there are representative contexts between the settled concepts and knowledge engineering is more acknowledged than knowledge modeling. In the following chapter the settled subjects must be defined and made distinguishable.

\section{ANALYSIS AND DISCUSSIONS}

At first the terms knowledge engineering and knowledge modeling will be investigated by analyzing the founded articles and their definitions. Afterwards the major differences between DSS and ES will be investigated. Consequently based on these descriptions the areas of application where knowledge models are used for ADMS will be described. In particular it will be examined if there are existing researches on the subject of large software development projects.

\section{A. Knowledge Modeling vs. Knowledge Engineering}

Knowledge engineering and knowledge modeling are used by most of the authors in a similar manner depending on the target domain [3], [4], [5], [6], [7], [8], [9], [10], [11] and [12]. Chan et al. considered in [13] the process of knowledge acquisition and modeling for structuring the knowledge base of an ES. In fields of product redesign and mechanical engineering, an ontology based knowledge model and reuse approach was studied by Liu et al. [7]. Here an ontology modeling method was considered for solving redesign problems based on an existing knowledge design that must be used in an effective and efficient way. According to Mendis et al. [8] knowledge modeling addresses languages, tools, techniques and methods to develop abstract models of some target domain or problem solving behavior. Some examples for tools and methods, that are serving to structure and represent knowledge, are semantic nets, topic maps or ontologies. A semantic net constitutes concepts and their relations with the aid of equivalent structures in terms of a graph with nodes and edges [15]. Topic maps, also called as knowledge maps, consist of subjects (topics, associations and occurrences) that are depicted in a human way of knowledge processing. Their description as XML enables for topic maps to be machine-readable. In the first place topic maps serve to a better navigation and search in internet resources and also for exchanging of metadata [16]. According to Chan [3] ontology offers a set of concepts and terms to describe a domain and those terms are used by knowledge bases for representing true about some real or imaginary world. It is a symmetric representation of knowledge with mechanism of inheritance to generate an ambiguous communication, for instance based on the Web Ontology Language.

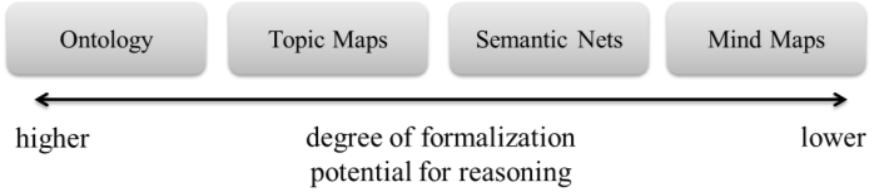

Fig. 2. Tools for knowledge modeling

According to [17] Fig. 2 shows the described tools dependent on their respective degree of formalization and thus their potential for reasoning. As brainstorming tool mind map symbolizes at this point just an arbitrary description of information in a specific use case. 
Knowledge modeling is also used in the domain of intelligent personalization of cognitive learning activities as Ramirez et al. [11] described. Here it is demonstrated how to associate main body units of knowledge to previous and future learned knowledge referring to basic of semantic net. Chan [3] defines knowledge models as problem-solving models of real world application domains for increasing effectiveness of knowledge based systems. In addition to developing effective knowledge bases, knowledge models serve to enable sharing and reuse of knowledge. Thus modeling knowledge bases can be used for structuring their information, data and associations by using methods of ontology and additionally generate sharing and reuse knowledge.

Chan [3] speaks of knowledge engineering as "process of eliciting expertise, organizing it into a computational structure, and building knowledge bases. This is a process that addresses directly internal information processing mechanisms and processes of human experts." Furthermore Chan issues that ontology engineering is deemed to be the successor of knowledge engineering. Ontology engineering shall improve a long-term efficiency of a knowledge based system development process, even in distributed environments. The authors Matta et al. [14] and Guaglianone et al. [5] describe knowledge engineering likewise for acquiring and modeling knowledge and for developing ES. It can be adapted to answer to aims of knowledge management. Hall [6] attends to the question concerning knowledge engineering in which way knowledge has to be integrated into a computer system. The knowledge in engineering design varies from that one in science. In particular modeling knowledge in science includes topics of decision making, reasoning, problem solving and the nature of thought itself. Whereas engineering knowledge for instance in an ES involves heuristics, theories, tools and techniques.

Ontology engineering as machine-readable method for modeling ontology-based knowledge bases to develop ES needs to be analyzed in more detail. According to [17] and [18] an ontology model is a purposeful created engineering artifact that serves for extensive declarative description of decision making models to reduce false reasoning and increase useful automatic interpretations and processing. Thereby it is difficult to afford a temporal stability of the ontology and most of all in cases of great and various ontologies to have a consistent formality and integrity of modeling. Moreover the scalable reasoning in ontology engineering is still not investigated for instance as an abstract model.

There are a range of tools for constructing ontologies for instance to model products and processes or to manage ontologies. Ontology maps attempt to automatically manage different ontologies for the purpose of information integration. However there also is given a lack of consistent formality and integrity of modeling [18].

The subject 'expert system' is still used in combination with knowledge engineering or ontology engineering. In the following it has to be defined where are the differences between DSS and ES.

\section{B. Decision Support System}

First time it was spoken about DDS was introduced by Morton [19] in terms of managing decision systems. Fick and Sprague [20] define a DSS as computerized planning and information system that presents useful information from raw data, documents or personal knowledge for helping to solve problems and make decisions, sometime in a graphical way. Based on comprehensive databases and settled rules such a knowledge-based system for decision making are applied to analyze, plan, budget as well as calculate data for strategic activities. According to [21], [22] and [23] DSS are often used for clinical and medical diagnoses and therapy decisions. Lu et al. [24] developed a prototype of a multi-objective group decision support system that addresses interactive applications for decision making by a group of participants which are not staying at the same place. Another group decision support system was used for railway construction decisions by Sheng et al. [25]. In most of these fields of application for DSS a simple decision tree was evolved to set the rules and thus to aggregate information or knowledge for presenting.

When a system is used to generate new knowledge, it is usually an ES, not a DDS. So the main difference between DSS and ES is in the fact of acquiring new knowledge, for instance an ES with a well modeled ontology-based knowledge base or an ES with a self-learning component such as artificial neural network.

\section{Expert System}

According to Russell et al. [26] "an expert system can be defined as an intelligent system that can mimic some part of human intelligence". To develop an artificial intelligence system it needs to be identified how intelligent behavior of human experts can be replicated by a computer system [27], [31]. ES are built by acquiring the knowledge from human experts and coding it into a machine-readable form. In combination with inference rules, e.g. cause/effect, situation/ action, if-then-else, it is used for problem solving, decision making, designing, planning, monitoring, diagnosing and training activities [27]. The literature has reported that people have difficulty in solving multi-dimensional problems of unknown issues fastest possible. In these cases human cognition reaches their limits [28]. An ES bunches expert and experience knowledge and supports decision makers in problem solving as a human expert of each specific domain at any time.

Guan et al. [29] also describe a weakness of traditional ES. It lies in the fact that there could be a lack of completeness in terms of covering various types of different knowledge (expert knowledge and knowledge acquisition), for instance in his field of application 'financial internal control systems'. According to Chan [3] constructing an ES is a knowledge intensive process and knowledge engineering or modeling is often adopted for building the knowledge base. Accordingly the modeled knowledge base and its continuous enhancement by knowledge acquisition, using the ES, is one of the most important points. 
As said by Tian et al. [30] and Qian et al. [32] artificial neural networks combined with knowledge bases can be used for developing ES and could give the possibility to construct a self-learning ES. An artificial neural network is, in accordance with [32], "a collection of basic units to compute a non-linear function of their input. Every input has an assigned weight that determines [its] impact [...] on the overall output of the node." Thus an ES is able to access a knowledge base that will be trained by artificial neural networks based on user input as well as the acquired and expert knowledge.

Fig. 3 illustrates in a simple way components and relations of ES and the acquiring knowledge process. Also it shows the relationship between knowledge modeling, knowledge engineering and ontology engineering. Decision makers can use an ES for solving problems. Based on rules and inference mechanism an inference system communicates to the knowledge base that can be modeled with methods of ontologies engineering. Knowledge bases of ES consist human-specific domain knowledge (expert knowledge) as well as experience knowledge, which is acquired from the ES itself. Artificial neural networks can be included in developing the knowledge base and for training the system. As result represented knowledge is made based on the inference system combined with the ontology-based knowledge base and/or for instance on the basis of artificial neural networks, as [30] investigated.

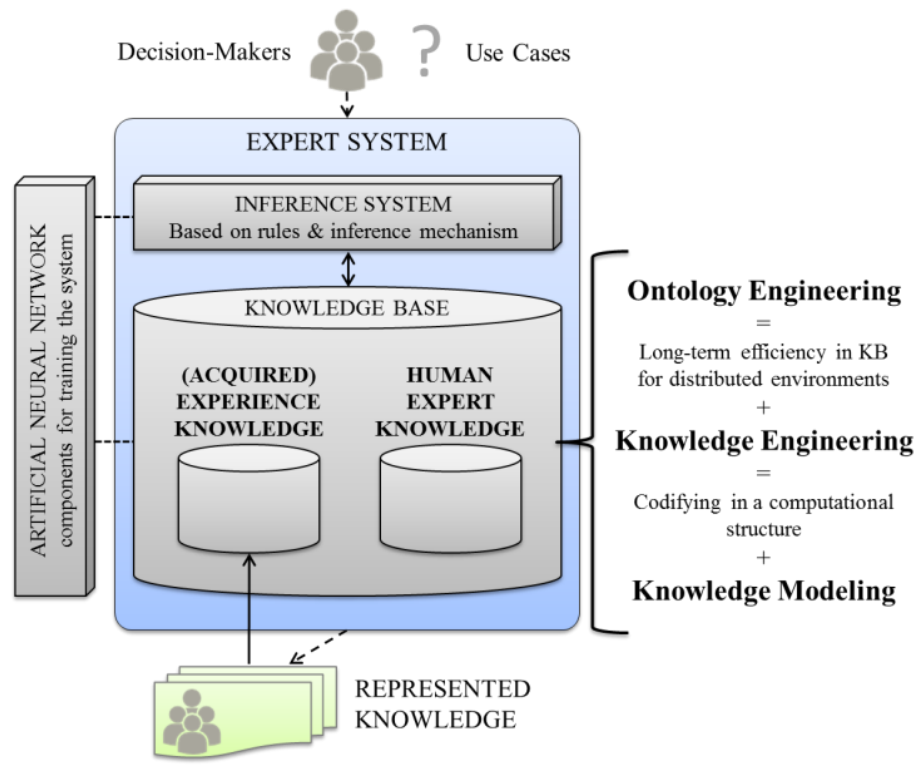

Fig. 3. Components and relations of Expert Systems

As described above inference methods and knowledge base provide the foundation of ES. Major problem of splitting these components is differentiating between human readable illustrating of knowledge and machine learning creating of knowledge. A model that combines visualization knowledge and inference mechanism would serve as better approach for building knowledge bases in ES.

\section{Fields of application}

The literature review has shown that DSS and ES are often used in the domain of medicine and therapy. Furthermore decision making systems are integrated in control or detecting failure systems for example in finance. Also in the fields of economics such as analyzing customer needs or designing industrial or service products ES are increasingly used. During the study of the identified literature it was not found any ES or DSS within the subject of large software development projects and their toolsets. These listed fields of application are just examples and no claim is raised on completeness.

\section{CONCLUSION}

Elaboration of this paper has shown that the manner of modeling knowledge bases of any decision making system is an important step to develop an ES. The tools for knowledge modeling are different dependent on their potential for reasoning. Ontology engineering is the successor of knowledge engineering and enables long-term efficient knowledge within the knowledge base. The limits of ontology engineering lie in combining various ontologies and creating a consistent formality and integrity of modeling as well as scalable reasoning. Artificial neural networks combined with ontology engineering and topic maps as methods for modeling a knowledge base of an ES can be appointed to build a selflearning ADMS.

By the example of choosing the ideal toolset in large software development projects, that may consist of project management, development or administration tools, an abstract knowledge model can be investigated and hence to fill the gaps of ontology engineering additionally. For this reason techniques of ontology engineering and artificial neural networks should be combined for developing a self-learning human-readable ES in the future.

\section{ACKKNOWLEDGMENTS}

We acknowledge support for the Article Processing Charge by the German Research Foundation and the Open Access Publication Fund of the Technische Universität Ilmenau.

\section{REFERENCES}

[1] F.F. Fuessl, J. Ciemala, "Variable Factors of Influence in Product Line Development", Computer Software and Applications Conference Workshops (COMPSACW), Vasteras, Sweden, 21-25 July, 2014, pp. 390-395

[2] J. Webster, R.T. Watson, "Analyzing the Past to Prepare for the Future: Writing a Literature Review", MIS Quarterly Vol. 26 No. 2, pp. xiiixxiii, 2002

[3] C.W Chan, "Cognitive informatics: a knowledge engineering perspective”, Cognitive Informatics, pp. 49-56, 2002

[4] S. Ghoniemy, O.H. Karam, "Semantic Network-based Digital Optical Computer Algorithms for Expert Systems and Massively Parallel Knowledge Base Applications", Journal of Next Generation Information Technology; Vol. 4 Issue 5, pp.133-146, 2013

[5] M.T. Guaglianone, R. Guarasci, N. Matta, J. Cahier, A. Benel, "Comparison and evaluation of knowledge modeling techniques towards the definition of a global approach: MNEMO (Methodology for kNowledgE acquisition and MOdelling)", Computer Sciences and Convergence Information Technology (ICCIT), pp. 777-782, 2011

[6] J.G. Hall, "Engineering knowledge engineering", Expert Systems: International Journal of Knowledge Engineering and Neural Networks, Vol 29(5), pp. 427-428, 2012

[7] X. Liu, D. Rosen, Z. Yu, "Ontology based Knowledge Modeling and Reuse Approach in Product Redesig", Information Reuse and Integration (IRI), pp. 270-273, 2010 
[8] D.S.K. Mendis, A.S. Karunananda, U. Samaratunga, U. Ratnayake, "Tacit knowledge modeling in Intelligent Hybrid systems", Industrial and Information Systems - ICIIS, pp. 279-284, 2007

[9] I. Mistrík, S. Beecham, I. Richardson, A. Avritzer, "Knowledge engineering in global software development environments", Expert Systems: International Journal of Knowledge Engineering and Neural Networks, Vol 31(3), pp. 232-233, 2014

[10] N. Moawad, K. Liu, M. El-Helly, "Integrating Activity Theory and Semiotics as Knowledge Elicitation Technique", Software Engineering, Artificial Intelligence, Networking and Parallel/Distributed Computing (SNPD), pp. 137-142, 2013

[11] C. Ramirez, B. Valdes, "Memory Map of a Knowledge Representation model used for intelligent personalization of learning activities sequences", Cognitive Informatics \& Cognitive Computing, pp. 423431, 2011

[12] X. Wu, H. Chen, G. Wu, J. Liu, Q. Zheng, X. He, A. zhou, Z. Zhao, B. Wei, Y. Li, Q. Zhang, S. Zhang:, "Knowledge Engineering with Big Data", Intelligent Systems, IEEE, pp. 1-19, 2015

[13] C.W. Chan, Y. Peng, L. Chen, "Knowledge acquisition and ontology modeling for construction of a control and monitoring expert system", International Journal of Systems Science, 33(6), pp. 485-503, 2002

[14] N. Matta, J.L. Ermine, G. Aubertin, J.Y. Trivin, "Knowledge capitalization with a knowledge engineering approach: the MASK method", Knowledge management and organizational memories, R. Dieng-Kuntz,N. Matta, Kluwer Academic Publishers, 2002

[15] T. Berners-Lee, J.A. Hendler, O. Lassila, "The Semantic Web", Scientific American, 284(5), pp. 34-43, 2001

[16] S. Pepper, "The TAO of Topic Maps" http://www.ontopia.net/ topicmaps/materials/tao.html, 2002

[17] C. Brewster, Y. Wilks, "Ontologies, Taxonomies, Thesauri: Learning from Texts", Deegan, M. (Hrsg.): The Use of Computational Linguistics in the Extraction of Keyword Information, Proceedings from Digital Library Content Workshop, Centre for Computing in the Humanities, 2004

[18] M. Hepp, "Possible Ontologies: How Reality Constrains the Development of Relevant Ontologies", IEEE Internet Computing 11(1), pp. $90-96,2007$

[19] M.S.S. Morton, "Management Decision Systems: Computer Based Support for Decision Making", Division of Research, Harvard University, Cambridge, MA, 1971
[20] G. Fick, R.H. Sprague, "Decision support systems: issues and challenges", Pergamon Press, Oxford, 1980

[21] D. Popescu, D. Lăptoiu, A. Hadăr, "Intelligent collaborative platform for development of personalized surgical orthopedic guides", Key Engineering Materials, Vol. 638, p303-309, 2014

[22] A. Oguntimilehin, A.O. Adetunmbi, K.A. Olatunji, "A Machine Learning Based Clinical Decision Support System for Diagnosis and Treatment of Typhoid Fever", International Journal of Advanced Research in Computer Science and Software Engineering, Vol 4,Issue 6, p961-969, 2014

[23] J. Colloc, L. Bouzidi, "A Framework for Clinical Decision Making and Medical Experience Storing”, ECIS 2000 Proceedings, Paper 125

[24] J. Lu, M. Quaddus, "A Prototype of Multi-Objective Group Decision Support System with a Group AggregationMethod base", ACIS 2001 Proceedings, Paper 48

[25] Q. Sheng, Z. Lei-shan, Y. Yi-xiang, "A web-based distributed group decision support system for railway construction organization", Informatics in Control, Automation and Robotics (CAR), pp. 362-365, 2010

[26] S. Russell, P. Norvig, "Artificial Intelligence: A Modern Approach", 3rd Ed., Prentice Hall, 2010

[27] G. Hwang, C. Chen, P. Tsai, C. Tsai, "An expert system for improving web-based problem-solving ability of students", Expert Systems with Applications, Vol. 38 Issue 7, pp.8664-8672, 2011

[28] D. Nguyen, D.S. Kira, "Designing Optimal Knowledge Base for Integrated Neural Expert Systems", AMCIS 2000 Proceedings, Paper 89

[29] J. Guan, A.S. Levitan, "A Model for Investigating Internal Control Weaknesses", Communications of the Association for Information Systems: Vol. 31, Article 3, 2012

[30] T. Tian, M. Zhu, B. Zhang, "An artificial neural network-based expert system for network topological error identification", Neural Networks 1995, Vol.2, pp. 882-886

[31] A. Kulinich, "Computer systems for cognitive maps simulation: Approaches and methods", Automation \& Remote Control. Vol. 73 Issue 9, p1553-1571, 2012

[32] F. Qian, L. Xu, "Improving customer satisfaction by the expert system using artificial neural networks", Intelligent Control and Automation 2008, pp. 8303-8306 